\title{
Mesenteric lymphadenitis caused by Yersinia enterocolitica
}

\author{
Justyna Zińczuk¹, Piotr Wojskowicz², Joanna Kiśluk³, Dawid Fil'4, Andrzej Kemona², Jacek Dadan² \\ ${ }^{1}$ Department of General Pathomorphology, Medical University of Bialystok, Bialystok, Poland \\ ${ }_{2}^{2} 1^{\text {st }}$ Clinical Department of General and Endocrine Surgery, Medical University of Bialystok, Bialystok, Poland \\ ${ }^{3} 2^{\text {nd }}$ Clinical Department of General and Gastroenterological Surgery, Medical University of Bialystok, Bialystok, Poland \\ ${ }^{4}$ Department of Histology and Embryology, Medical University of Bialystok, Bialystok, Poland
}

Prz Gastroenterol 2015; 10 (2): 118-121

DOI: $10.5114 /$ pg.2014.47504

Key words: yersiniosis, infection, mesenteric lymph nodes.

Address for correspondence: Justyna Zińczuk MD, Department of General Pathomorphology, Medical University of Bialystok, 13 Waszyngtona St, 15-269 Bialystok, Poland, phone: +48 663480 618, e-mail: justyna.zinczuk@umb.edu.pl

\begin{abstract}
Yersiniosis is an acute or chronic, zoonotic disease caused by infection of Gram-negative rods Yersinia enterocolitica. It can be transmitted by the consumption of originally contaminated food products (pork, unpasteurized milk) or secondarily contaminated with animal or vegetable products. The clinical picture of infection may have a variable course is related to the age and physical condition of the patient, or pathogenic properties of microorganisms. Infection caused by $Y$. enterocolitica can occur in different clinical forms: food poisoning, colitis, mesentric lymphadenitis, erythema nodosum, arthritis, pharyngitis, pneumonia, meningitis, sepsis. The aim of this study was to present a rare case of infection with $Y$. enterocolitica mesenteric lymph nodes coexistent with appendicitis.
\end{abstract}

\section{Introduction}

Yersiniosis is an acute or chronic, zoonotic disease caused by infection of Gram-negative rods Yersinia enterocolitica. The natural reservoirs of these bacteria are wild and household animals (pigs, cows, horses, sheep, goats, dogs, cats, and rabbits) as well as birds (turkeys, ducks, geese, pigeons, pheasants, and canaries). The source of the infection is mainly food and water contaminated by excrement of sick animals. The consumption of originally contaminated food products (undercooked pork, unpasteurised milk) or secondarily contaminated animal or vegetable products, leads to the infection. In cases of insufficient hygiene, the faecal-oral transmission of Yersinia is also possible (contact with contaminated animal excrement). Infection may also be initiated during blood transfusion from patients with asymptomatic bacteraemia or by contaminated dialysate [1, 2].

After reaching the human body and proliferating in the lumen of the gastrointestinal tract, Yersinia penetrates the $M$ cells of small intestine mucosa. This process causes the endocytosis of bacteria, their secretion to the mucosa stroma, and transportation to the lymph follicles.

Depending on the state of immune system of the carrier and the pathogenic properties of the strain, the bacteria may get into lymph nodes of the mesentery through lymphatic vessels and cause their inflammation. In consequence, it may lead to general infection of blood and haematosepsis [3].

The clinical picture of infection may have a variable course, which is related to the patient's age and the condition of the patient's immune system, or the pathogenic properties of the microorganisms. The most common forms of the disease are enteric and similar to appendicitis. Enteric forms primarily include enteritis and colitis with the following symptoms: secretory diarrhoea (frequently with blood and mucus), fever, abdominal pain, and rarely emesis. Those symptoms occur most frequently in children under 5 years old. Symptoms similar to appendicitis include adenitis mesenterica (lymphadenitis mesenterialis) and inflammation of the distal part of the small intestine and cecum. Those disorders affect mainly older children and adults. Adenitis mesenterica may imitate symptoms of the ap- 
pendicitis (fever, nausea, and abdominal pain located in right lower quadrant of the abdomen, near the right hip bone). Other, rarely observed symptoms include severe haematosepsis with high mortality rate and suppurative inflammations with different localisation: pharyngitis, pneumonia, meningitis, liver, kidneys, lungs and splenic abscesses, arthritis, osteomyelitis, dermatitis, urinary tract infection, and erythema nodosum [4].

The basis for recognition of yersiniosis is microbiological examination of material taken from the patients: faeces, blood, fluid taken from abscesses, other body fluids (e.g. peritoneal liquor), exudative synovial fluid, pharyngeal swab, swab from the lumen of appendicitis, and swab from intraoperatively taken mesenteric lymphatic node. In order to confirm infection, a serological examination is needed: tube agglutination test, passive haemagglutination test or commonly applied enzyme-linked immunosorbent assay (ELISA), which allows antibodies IgM, IgG and IgA to be observed in serum directed against antigens or bacterial proteins. Laboratory tests of patients with the suspicion of Y. enterocolitica show decreased levels of inflammatory process indicators such as leucocytosis, erythrocyte sedimentation rate, and C-reactive protein. The most significant role for patients after surgical treatment is played by histological evaluation of removed tissues. Non-specific microscopic image shows ulceration of intestinal mucosa, hyperplasia of lymphoid follicles, and infiltration of mononuclear cells with a predominance of histiocytes.

The above-described pathology is presented according to the rarity of the coexistence of appendicitis with adenitis mesenterica caused by $Y$. enterocolitica, and diagnostic difficulties connected with non-specific symptoms.

\section{Case report}

A 19-year-old man was admitted to the $1^{\text {st }}$ Department of General and Endocrinological Surgery, Medical University of Bialystok because of constantly escalating abdominal pain during the previous 3 days. Initially the pain embraced the whole abdominal cavity (mainly the epigastrium and middle abdominal region). On the admission day the pain was sensed in the right lower quadrant of the abdomen, near the right hip bone. The patient's body temperature was raised $\left(37.5^{\circ} \mathrm{C}\right)$. Physical examination of the abdomen revealed slight distention, slow peristalsis, abdominal guarding, and Blumberg's sign (rebound tenderness) in the right hypogastric region. Biochemical examinations revealed leucocytosis with "left shift" (leukocytes $15.1 \times 10^{3} \mu \mathrm{l}$, band cells 19 , multilayer granulocytes 60 , acidophiles 2 , lymphocytes 9 , monocytes 10 ) and anaemia (erythro- cytes $-3.92 \times 10^{6} / \mu \mathrm{l}$, haemoglobin $-11.5 \mathrm{~g} / \mu \mathrm{l}$, haematocrit $-35.5 \%$ ) as well as decreased concentration of C-reactive protein - $93.8 \mathrm{mg} / \mathrm{dl}$. Abdominal ultrasonography showed a small amount of fluid between the intestinal loops in the right lower quadrant of the abdomen (Figure 1) and numerous enlarged mesenteric lymph nodes of 20-45 mm (Figure 2).

The patient was qualified for surgical treatment ad hoc. During the operation a large amount of ambercoloured liquid was observed in the abdominal cavity as well as appendicitis, numerous enlarged $(1-2 \mathrm{~cm})$ mesenteric lymph nodes, and Meckel's diverticulum with broad base without inflammation, located approximately $50 \mathrm{~cm}$ from the ileocecal valve. Appendectomy was performed along with biopsy of mesenteric lymph

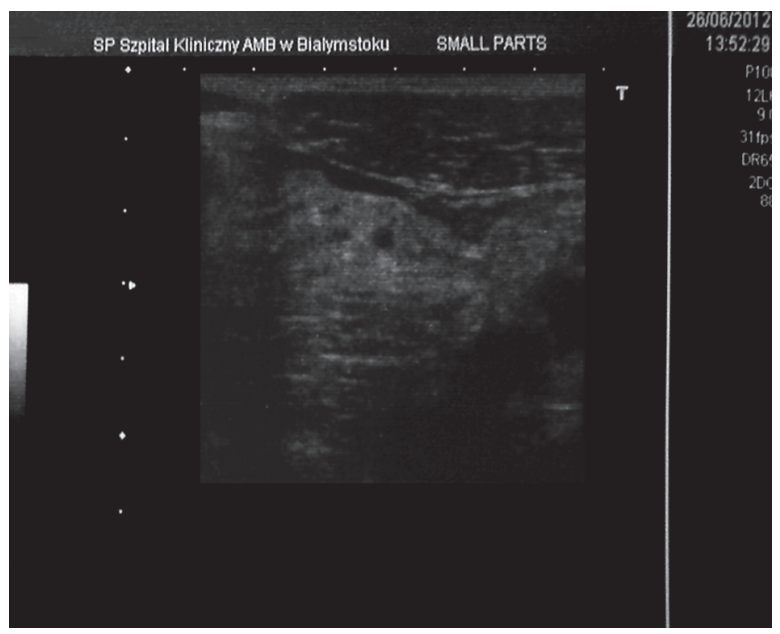

Figure 1. Preoperative USG of abdominal cavity. Fluid between intestinal loops in right lower quadrant of the abdomen, near the right hip bone

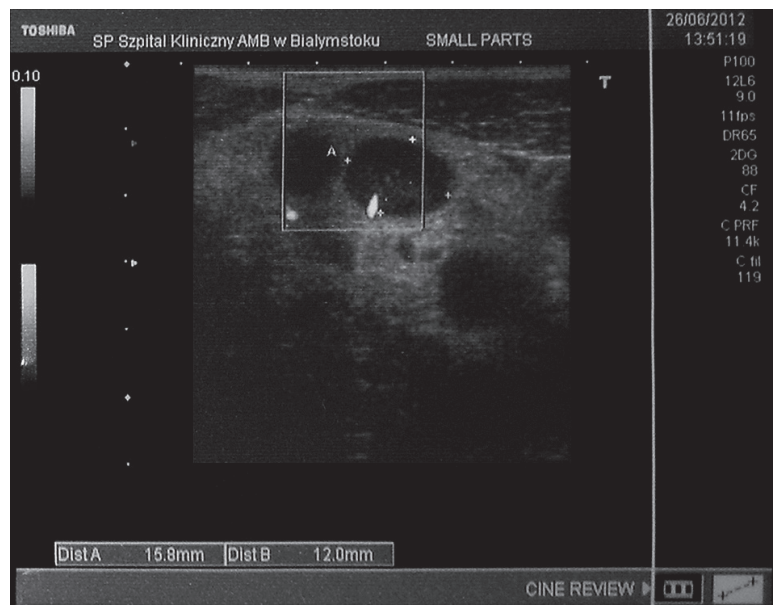

Figure 2. Preoperative USG of abdominal cavity. Enlarged mesenteric lymph nodes 


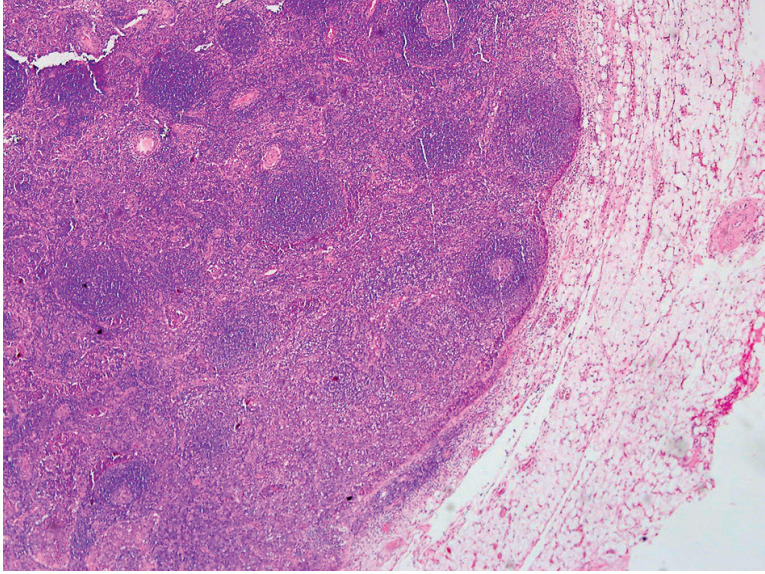

Figure 3. Lymph node with clearly visible lymph follicles. Magnification 4x, H + E staining

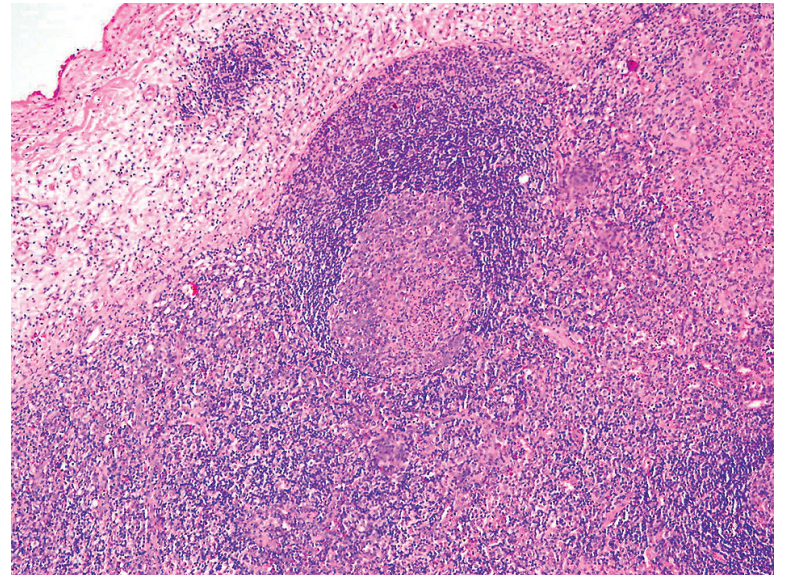

Figure 4. Lymph follicle. In the middle the blast with small infiltration from neutrophilic granulocytes and creation of microabscesses. Magnification $10 \times, \mathrm{H}+\mathrm{E}$ staining

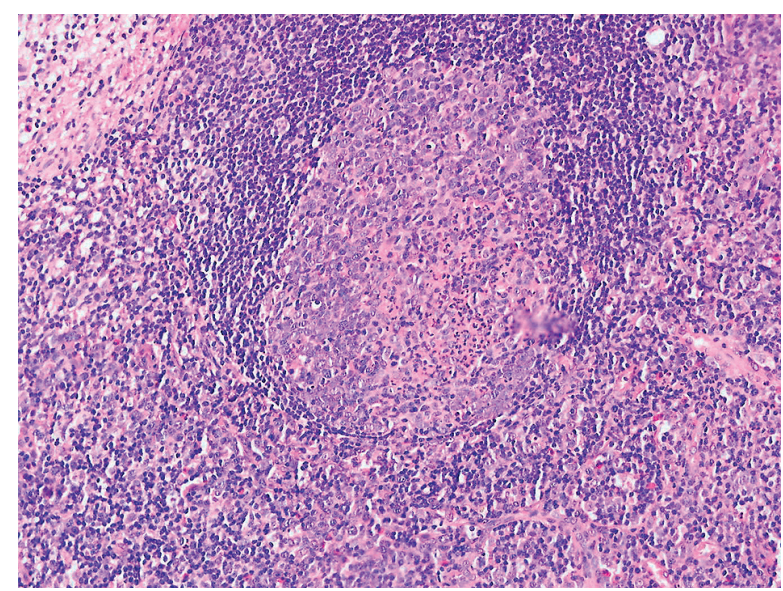

Figure 5. Lymph follicle. In the middle the blast with small infiltration from neutrophilic granulocytes and creation of microabscesses. Magnification $20 \times, \mathrm{H}+$ E staining

node. Postoperative complications were not observed After 3 days the patient was dismissed home in good general state and with recommendation of further antibiotic therapy (ciprofloxacin $2 \times 500 \mathrm{mg}$ p.o.) and control visit in the Surgical Outpatient Clinic 7 days after the surgery. During the control visit the patient felt well, the wound was healed, and the stitches were removed. Once again, ultrasonography was performed and revealed a hint of fluid between the intestinal loops and a large amount of hypoechogenic lymph nodes of $39 x$ $18 \mathrm{~mm}$. As a result, continuation of antibiotic therapy was recommended. Histopathological examination of removed appendicitis revealed phlegmonous appen-

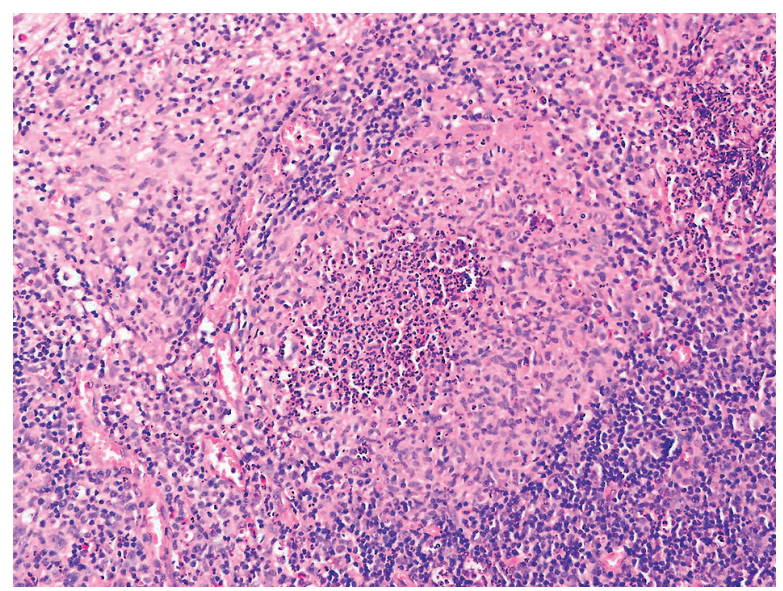

Figure 6. Lymph follicle destroyed by inflammatory process in enlarged blast and multiple microabscesses. Magnification 20x, H + E staining

dicitis, and, in mesenteric lymph nodes, significant hyperplasia of lymphoid follicles with enlargement of proliferation centres with visible infiltration from multilayer neutrophilic granulocytes was observed (Figures 3-5). What is more, in histopathological image microabscesses were observed (Figure 6), which suggested adenitis mesenterica caused by $Y$. enterocolitica. Final recognition of yersiniosis was confirmed by serological test performed with ELISA. This examination of serum was conducted in order to find antibodies for Yersinia outer proteins and purified $\mathrm{O}$ antigens (lipopolysaccharide) obtained from microbes from different serological groups of $Y$. enterocolitica. The assay of antibody titre 
for Yersinia outer proteins in different classes of immunoglobins gave the following results: IgA - 49 (negative < 15), IgG - 1235 (negative < 75), and IgM - 204 (negative $<15)$. The above results confirmed our previous hypothesis.

\section{Discussion}

Infection with $Y$. enterocolitica is a rare but very important medical issue all over the world. Infections occur mainly in highly developed and developing countries. In Poland the incidence of yersiniosis is constantly growing. In 2005, 135 cases were noticed; 2 years later 233 cases were recognised including 182 cases of intestinal yersiniosis. In 2010, the amount of infected patients was 288 , which confirms an increasing tendency of incidence of the disease. However, the incidence coefficient in Poland is significantly lower (0.62 per 100 thousand citizens) in comparison to other European Union countries (2.6 per 100 thousand citizens). The aetiology is mainly connected with difficulties in diagnosis according to the non-specific character of the infection and multiple cross reactions [5].

Clinical differentiation of yersiniosis symptoms is huge: from spontaneously resolving diarrhoeas to severe infections with bacteraemia. The reasons include age, physical state of the patient, and pathogenic factors of bacteria, which depend on its strain [6].

The most common and diagnostically difficult type is intestinal yersiniosis, as well as the type similar to appendicitis in children and young adults. The symptoms include diarrhoea, emesis, and sometimes fever and pain located in middle abdominal region and right hypogastric region, which are connected with inflammation and enlarged mesenteric lymph nodes, especially in the ileocecal area $[7,8]$. In the majority of cases, the pain initially embraces the whole abdominal cavity or umbilical area and its character is diffuse. During the following hours or days it is sensed in the right hypogastric region. During physical examination, some patients demonstrate hypertonia in the right hypogastric area and even some symptoms indicating peritonitis. Laboratory tests usually show elevated erythrocyte sedimentation rate, increase of C-reactive protein, and leucocytosis. The majority of patients who do not suffer from diarrhoea are treated surgically according to the symptoms typical for appendicitis. During the operation, unchanged (or rarely inflammatory) appendix and enlarged ileocecal lymph nodes with inflammation are observed along with increased amount of clear, amber-coloured fluid in the peritoneal cavity. In the above case, abdominal pain in right lower quadrant of the abdomen, near the right hip bone, enlargement of lymph nodes, and presence of amber-coloured fluid in the abdominal cavity suggested infection with Yersinia, which was confirmed in histopathological examination of removed lymph node and serological tests.

\section{Conclusions}

The frequency of occurrence of yersiniosis among patients with pain in the right hypogastric area and suspected appendicitis oscillates around $2-23 \%$. It is recommended that physicians provide for the possibility of substantial but still rare infection with $Y$. enterocolitica in diagnostics of non-specific symptoms.

\section{Conflict of interest}

The authors declare no conflict of interest.

\section{References}

1. Talarek E, Rastawicki W, Banasiuk M, et al. Clinical picture and diagnostics problems of yersiniosis in children [Polish]. Ped Wsp Gastr Hepat Żyw Dziecka 2009; 11: 9-12.

2. Janowska M, Jędrzejewska B, Janowska J. Yersiniosis - a new challenge for contemporary medicine [Polish]. Med Og Nauk Zdr 2012; 18: 257-60.

3. Galindo CL, Rosenzweig JA, Kirtley ML, et al. Pathogenesis of Y. enterocolitica and Y. pseudotuberculosis in human yersiniosis. J Pathog 2011; 2011: 182051. DOI: 10.4061/2011/182051.

4. Jagielski M, Rastawicki W, Kałużewski S, et al. Jersinioza niedoceniana choroba zakaźna [Polish]. Przegl Epidemiol 2000; 56: 57-64.

5. Furman S, Sadkowska-Todys M. Yersiniosis in Poland in 2010. Przegl Epidemiol 2012; 66: 249-53.

6. Perdikogianni C, Galanakis E, Michalakis M, et al. Yersinia enterocolitica infection mimicking surgical condition. Pediatr Surg Int 2006; 7: 589-92.

7. Fuchizaki U, Machi T, Kaneko S. Clinical challenges and images in GI. Yersinia enterocolitica mesenteric adenitis and terminal ileitis. Gastroenterology 2006; 131: 1379-81.

8. Coussi A, Athanassiou M, Tsantali C, et al. Yersinia enterocolitica mesenteric adenitis suspected by ultrasound examination. Eur J Pediatr 1994; 153: 299.

Received: 22.01 .2013

Accepted: 29.01.2013 